\title{
Fuzzy fixed point theorems for fuzzy mappings via $\beta$-admissible with applications
}

\author{
Supak Phiangsungnoen ${ }^{1}$, Wutiphol Sintunavarat ${ }^{2}$ and Poom Kumam ${ }^{1 *}$
}

\author{
*Correspondence: \\ poom.kum@kmutt.ac.th \\ 1 Department of Mathematics, \\ Faculty of Science, King Mongkut's \\ University of Technology Thonburi \\ (KMUTT), 126 Pracha Uthit Rd., Bang \\ Mod, Thung Khru, Bangkok 10140, \\ Thailand \\ Full list of author information is \\ available at the end of the article
}

\begin{abstract}
The object of this paper is to utilize the notion of $\beta$-admissible in the sense of Mohammadi et al. (Fixed Point Theory Appl. 2013:24, 2013) to prove a fuzzy fixed point theorem and present some corollaries. We also give illustrative examples which demonstrate the validity of hypotheses of our results and applications to fuzzy fixed points for fuzzy mappings in partially ordered metric spaces are studied.
\end{abstract}

Keywords: Fuzzy set; Fuzzy mapping; Fuzzy fixed point; $\beta$-admissible mappings

\section{Introduction}

In real world problems, fuzzy concepts were used to modeled mathematically for the purpose of automation, like regulation, production control and many others. The concept of fuzzy set was introduced by Zadeh [1]. Several researches were conducted on the generalizations of the concept of a fuzzy set. Heilpern [2] introduced the concept of fuzzy contraction mappings which maps from an arbitrary set to a certain subfamily of fuzzy sets in a metric linear space $X$. He also proved the existence of a fuzzy fixed point theorem which is a generalization of Nadler's [3] fixed point theorem for multivalued mappings.

In 2001, Estruch and Vidal [4] proved a fuzzy fixed point theorem for fuzzy contraction mappings (in the Heilperns sense) over a complete metric space. Afterward, many mathematicians [5-9] generalized the result given in [4]. Recently, Abbas and Turkoglu [10] established the existence of a fuzzy fixed point theorem for fuzzy mapping of a generalized contractive mapping on a complete metric space, which generalized and extended the results in [4] and many known results in $[2,6]$.

On the other hand, the concept of an $\beta$-admissible mapping was introduced by Samet et al. [11]. They also give the existence of fixed point theorems via this concept. Afterward, Asl et al. [12] extended the concept of $\beta$-admissible for single-valued mappings to multivalued mappings. Recently, Mohammadi et al. [13] introduced the concept of $\beta$ admissible for multivalued mappings which is different from the notion of $\beta_{*}$-admissible which has been provided in [12]. Recently, Azam et al. [14], obtained common fixed point theorems for Chatterjea fuzzy mappings on closed balls in a complete metric space. The investigation is based on the fact that fuzzy fixed point results can be obtained simply from the fixed point theory of mappings on closed balls.

In this paper, we prove a fuzzy fixed point for a generalized contractive fuzzy mapping on a complete metric space by using the concept of $\beta$-admissible mapping in sense of

(c) 2014 Phiangsungnoen et al.; licensee Springer. This is an Open Access article distributed under the terms of the Creative Commons Attribution License (http://creativecommons.org/licenses/by/2.0), which permits unrestricted use, distribution, and reproduction in any medium, provided the original work is properly cited. 
Mohammadi et al. [13]. We also give illustrative example which demonstrate the validity of hypotheses of our results. As an application, we deduce fixed point theorems in partially ordered metric spaces.

\section{Preliminaries}

In this section, we recall some basic definitions and preliminaries that will be needed in this paper.

Definition 1 ([1]). Let $X$ be a space of points with generic elements of $X$ denoted by $x$ and $I=[0,1]$. A fuzzy subset of $X$ is characterized by a membership function such that each element in $X$ is associated with a real number in the interval $I$.

Definition 2 ([2]). Let $(X, d)$ be a metric space and $A$ be a fuzzy set in $X$. If $X$ is endowed with topology, for $\alpha \in[0,1]$, the $\alpha$-level set of $A$, denoted by $[A]_{\alpha}$, is defined as

$$
[A]_{\alpha}=\{x: A(x) \geq \alpha\}
$$

where $\alpha \in(0,1]$ and for $\alpha=0$ we have

$$
[A]_{0}=\overline{\{x: A(x)>0\}},
$$

where $\bar{B}$ denotes the closure of the non-fuzzy set $B$.

Definition 3 ([15]). Let $X$ be a nonempty set. For $x \in X$, we write $\{x\}$ the characteristic function of the ordinary subset $\{x\}$ of $X$. For $\alpha \in(0,1]$, the fuzzy point $x_{\alpha}$ of $X$ is the fuzzy set of $\mathrm{X}$ given by

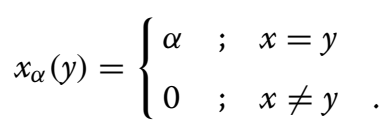

In the sequel, $I=[0,1],(X, d)$ and $I^{X}$ denote the metric space and the collection of all fuzzy subsets of $X$. For $A, B \in I^{X}$, a fuzzy set $A$ is said to be more accurate than a fuzzy set $B$ (denoted by $A \subset B$ ) if and only if $A x \leq B x$ for each $x$ in $X$, where $A(x)$ and $B(x)$ denote the membership function of $A$ and $B$, respectively. For $x \in X, S \subset X, A, B \in I^{X}$ and $\alpha \in[0,1]$, we define

$$
\begin{aligned}
d(x, S) & =\inf \{d(x, a) ; a \in S\}, \\
p_{\alpha}(x, A) & =\inf \left\{d(x, a) ; a \in[A]_{\alpha}\right\}, \\
p_{\alpha}(A, B) & =\inf \left\{d(a, b) ; a \in[A]_{\alpha}, b \in[B]_{\alpha}\right\}, \\
p(A, B) & =\sup _{\alpha} p_{\alpha}(A, B), \\
D_{\alpha}(A, B) & =H\left([A]_{\alpha},[B]_{\alpha}\right)=\max \left\{\sup _{a \in[A]_{\alpha}} d\left(a,[B]_{\alpha}\right), \sup _{b \in[B]_{\alpha}} d\left(b,[A]_{\alpha}\right)\right\}, \\
d_{\infty}(A, B) & =\sup _{\alpha} D_{\alpha}(A, B),
\end{aligned}
$$

and

$$
W_{\alpha}(X)=\left\{C \in I^{X}:[C]_{\alpha} \text { is nonempty and compact }\right\} .
$$

It is easy to see that $H$ is the Hausdorff metric on $W_{\alpha}(X)$ induced by the metric $d$. 
Definition 4 ([4]). Let $(X, d)$ be a metric space. $T: X \rightarrow I^{X}$ and $\alpha \in[0,1]$. A fuzzy point $x_{\alpha}$ in $X$ is called fuzzy fixed point of $T$ if $x_{\alpha} \subset T x$. If $\{x\} \subset T x$, then $x$ is called fixed point of $T$.

Definition 5 ([12]). Let $X$ be a nonempty set, $T: X \rightarrow 2^{X}$, where $2^{X}$ is a collection of nonempty subsets of $X$ and $\beta: X \times X \rightarrow[0, \infty)$. We say that $T$ is $\beta_{*}$-admissible if

$$
\text { for } x, y \in X, \quad \beta(x, y) \geq 1 \Longrightarrow \beta_{*}(T x, T y) \geq 1,
$$

where

$$
\beta_{*}(T x, T y):=\inf \{\beta(a, b): a \in T x \text { and } b \in T y\} .
$$

Definition 6 ([13]). Let $X$ be a nonempty set, $T: X \rightarrow 2^{X}$, where $2^{X}$ is a collection of nonempty subsets of $X$ and $\beta: X \times X \rightarrow[0, \infty)$. We say that $T$ is $\beta$-admissible whenever for each $x \in X$ and $y \in T x$ with $\beta(x, y) \geq 1$, we have $\beta(y, z) \geq 1$ for all $z \in T y$.

Remark 1. If $T$ is $\beta^{*}$-admissible mapping, then $T$ is also $\beta$-admissible mapping.

Example 1. Let $X=\mathbb{R}$ be endowed with the usual metric $d$. Define mappings $T: X \rightarrow$ $2^{X}$ and $\beta: X \times X \rightarrow[0, \infty)$ by

$$
T(x)=\left\{\begin{array}{l}
{[x, x+1], \text { if } x \geq 0} \\
(-\infty, 6 x], \text { if } x<0
\end{array}\right.
$$

and

$$
\beta(x, y)=\left\{\begin{array}{l}
1, \text { if } x, y \geq 0 ; \\
0, \text { if otherwise. }
\end{array}\right.
$$

Then $T$ is $\beta$-admissible.

\section{Fuzzy fixed point theorem}

In what follows, we give some well known lemma which is useful for the proof.

Lemma 1 ([2]). Let $(X, d)$ be a metric space, $x \in X$ and $A \in I^{X}$. For $\alpha \in[0,1]$, if $p_{\alpha}(x, A)=0$ and $[A]_{\alpha}$ is closed subset of $X$, then $x_{\alpha} \subset A$.

Now, we introduce the concept of $\beta$-admissible in sense of Mohammadi et al. [13] for fuzzy mapping.

Definition 7. Let $(X, d)$ be a metric space, $\beta: X \times X \rightarrow[0, \infty), \alpha \in[0,1]$ and $T: X \rightarrow$ $W_{\alpha}(X)$. A mapping $T$ is said to be $\beta$-admissible if for each $x \in X$ and $y \in[T x]_{\alpha}$, with $\beta(x, y) \geq 1$, we have $\beta(y, z) \geq 1$ for all $z \in[T y]_{\alpha}$.

Next, we prove the existence of fuzzy fixed point theorem for some generalized type of contraction fuzzy mapping. For our result, we let $\Psi$ be the family of non-decreasing functions $\psi:[0, \infty) \rightarrow[0, \infty)$ such that $\sum_{n=1}^{\infty} \psi^{n}(t)<\infty$ for each $t>0$. It is easy to see that for $\psi \in \Psi, \psi(t)<t$ for all $t>0$ and $\psi(0)=0$. 
Theorem 1. Let $(X, d)$ be a complete metric space, $\alpha \in[0,1]$ and $T$ be fuzzy mapping from $X$ to $W_{\alpha}(X)$. Suppose that there exist $\psi \in \Psi$ and $\beta: X \times X \rightarrow[0, \infty)$ such that

$$
\beta(x, y) D_{\alpha}(T x, T y) \leq \psi(M(x, y))+L \min \left\{p_{\alpha}(x, T x), p_{\alpha}(y, T y), p_{\alpha}(x, T y), p_{\alpha}(y, T x)\right\}
$$

for all $x, y \in X$, where $L \geq 0$ and

$$
M(x, y)=\max \left\{d(x, y), p_{\alpha}(x, T x), p_{\alpha}(y, T y), \frac{p_{\alpha}(x, T y)+p_{\alpha}(y, T x)}{2}\right\} .
$$

If the following condition holds,

(i) $T$ is $\beta$-admissible,

(ii) there exist $x_{0} \in X$ and $x_{1} \in\left[T x_{0}\right]_{\alpha}$ such that $\beta\left(x_{0}, x_{1}\right) \geq 1$,

(iii) if $\left\{x_{n}\right\}$ is sequence in $X$ such that $\beta\left(x_{n}, x_{n+1}\right) \geq 1$ and $x_{n} \rightarrow u$ as $n \rightarrow \infty$, then $\beta\left(x_{n}, u\right) \geq 1$,

(iv) $\psi$ is continuous,

then there exists $x \in X$ such that $x_{\alpha}$ is a fuzzy fixed point of $T$.

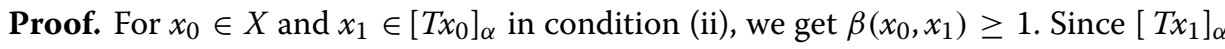
is nonempty compact subset of $X$, there exists $x_{2} \in\left[T x_{1}\right]_{\alpha}$, such that

$$
d\left(x_{1}, x_{2}\right)=p_{\alpha}\left(x_{1}, T x_{1}\right) \leq D_{\alpha}\left(T x_{0}, T x_{1}\right) .
$$

From (2) and the fact that $\beta\left(x_{0}, x_{1}\right) \geq 1$, we have

$$
\begin{aligned}
d\left(x_{1}, x_{2}\right) & \leq D_{\alpha}\left(T x_{0}, T x_{1}\right) \\
& \leq \beta\left(x_{0}, x_{1}\right) D_{\alpha}\left(T x_{0}, T x_{1}\right) \\
& \leq \psi\left(M\left(x_{0}, x_{1}\right)\right)+L \min \left\{p_{\alpha}\left(x_{0}, T x_{0}\right), p_{\alpha}\left(x_{1}, T x_{1}\right), p_{\alpha}\left(x_{0}, T x_{1}\right), p_{\alpha}\left(x_{1}, T x_{0}\right)\right\} \\
& \leq \psi\left(M\left(x_{0}, x_{1}\right)\right)+L \min \left\{p_{\alpha}\left(x_{0}, x_{1}\right), p_{\alpha}\left(x_{1}, x_{2}\right), p_{\alpha}\left(x_{0}, x_{2}\right), 0\right\} \\
& =\psi\left(M\left(x_{0}, x_{1}\right)\right) .
\end{aligned}
$$

By the same argument, for $x_{2} \in X$, we have $\left[T x_{2}\right]_{\alpha}$, which is a nonempty compact subset of $X$ and then there exists $x_{3} \in\left[T x_{2}\right]_{\alpha}$ such that

$$
d\left(x_{2}, x_{3}\right)=p_{\alpha}\left(x_{2}, T x_{2}\right) \leq D_{\alpha}\left(T x_{1}, T x_{2}\right) .
$$

For $x_{0} \in X$ and $x_{1} \in\left[T x_{0}\right]_{\alpha}$ with $\beta\left(x_{0}, x_{1}\right) \geq 1$, by definition of $\beta$-admissible, we get

$$
\beta\left(x_{1}, x_{2}\right) \geq 1 \text {. }
$$

From (1), (3) and (4), we have

$$
\begin{aligned}
d\left(x_{2}, x_{3}\right) & \leq D_{\alpha}\left(T x_{1}, T x_{2}\right) \\
& \leq \beta\left(x_{1}, x_{2}\right) D_{\alpha}\left(T x_{1}, T x_{2}\right) \\
& \leq \psi\left(M\left(x_{1}, x_{2}\right)\right)+L \min \left\{p_{\alpha}\left(x_{1}, T x_{1}\right), p_{\alpha}\left(x_{2}, T x_{2}\right), p_{\alpha}\left(x_{1}, T x_{2}\right), p_{\alpha}\left(x_{2}, T x_{1}\right)\right\} \\
& \leq \psi\left(M\left(x_{0}, x_{1}\right)\right)+L \min \left\{p_{\alpha}\left(x_{1}, x_{2}\right), p_{\alpha}\left(x_{2}, x_{3}\right), p_{\alpha}\left(x_{1}, x_{3}\right), 0\right\} \\
& =\psi\left(M\left(x_{1}, x_{2}\right)\right) .
\end{aligned}
$$

By induction, we can construct a sequence $\left\{x_{n}\right\}$ in $X$ such that, for each $n \in \mathbb{N}, x_{n} \in$ $\left[T x_{n-1}\right]_{\alpha}$ with $\beta\left(x_{n-1}, x_{n}\right) \geq 1$ and

$$
d\left(x_{n}, x_{n+1}\right) \leq \psi\left(M\left(x_{n-1}, x_{n}\right)\right),
$$


where

$$
\begin{aligned}
M\left(x_{n-1}, x_{n}\right) & =\max \left\{d\left(x_{n-1}, x_{n}\right), p_{\alpha}\left(x_{n-1}, T x_{n-1}\right), p_{\alpha}\left(x_{n}, T x_{n}\right), \frac{p_{\alpha}\left(x_{n-1}, T x_{n}\right)+p_{\alpha}\left(x_{n}, T x_{n-1}\right)}{2}\right\} \\
& \leq \max \left\{d\left(x_{n-1}, x_{n}\right), d\left(x_{n}, x_{n+1}\right), \frac{d\left(x_{n-1}, x_{n+1}\right)}{2}\right\} \\
& =\max \left\{d\left(x_{n-1}, x_{n}\right), d\left(x_{n}, x_{n+1}\right)\right\} .
\end{aligned}
$$

Hence,

$$
d\left(x_{n}, x_{n+1}\right) \leq \psi\left(\max \left\{d\left(x_{n-1}, x_{n}\right), d\left(x_{n}, x_{n+1}\right)\right\}\right)
$$

for all $n \in \mathbb{N}$. If there exists $n^{*} \in \mathbb{N}$ which $p_{\alpha}\left(x_{n^{*}}, T x_{n^{*}}\right)=0$, then from Lemma 1, we have $\left(x_{n^{*}}\right)_{\alpha} \subset T x_{n^{*}}$, that is $\left(x_{n^{*}}\right)_{\alpha}$ is a fuzzy fixed point of $T$. Therefore, we suppose that for each $n \in \mathbb{N}, p_{\alpha}\left(x_{n}, T x_{n}\right)>0$ and thus $d\left(x_{n-1}, x_{n}\right)>0$ for all $n \in \mathbb{N}$. So, if $d\left(x_{n}, x_{n+1}\right)>d\left(x_{n-1}, x_{n}\right)$ for some $n \in \mathbb{N}$, then from (5) and $\psi(t)<t$ for $t \in(0, \infty)$, we have

$$
d\left(x_{n}, x_{n+1}\right) \leq \psi\left(d\left(x_{n}, x_{n+1}\right)\right)<d\left(x_{n}, x_{n+1}\right)
$$

which is a contradiction. Therefore, we have

$$
\begin{aligned}
d\left(x_{n}, x_{n+1}\right) & \leq \psi\left(d\left(x_{n-1}, x_{n}\right)\right) \\
& \leq \psi\left(\psi\left(d\left(x_{n-2}, x_{n-1}\right)\right)\right) \\
& \vdots \\
& \leq \psi^{n}\left(d\left(x_{0}, x_{1}\right)\right) .
\end{aligned}
$$

Next, we will show that $\left\{x_{n}\right\}$ is a Cauchy sequence in $X$. Since continuous function $\psi$ is belong to $\Psi$, there exist $\varepsilon>0$ and positive integer $h=h(\varepsilon)$ such that

$$
\sum_{n \geq h} \psi^{n}\left(d\left(x_{0}, x_{1}\right)\right)<\varepsilon
$$

Let $m>n>h$. Using the triangular inequality, previous relation and (6), we have

$$
d\left(x_{n}, x_{m}\right) \leq \sum_{k=n}^{m-1} d\left(x_{k}, x_{k+1}\right) \leq \sum_{k=n}^{m-1} \psi^{k}\left(d\left(x_{0}, x_{1}\right)\right) \leq \sum_{n \geq h} \psi^{n}\left(d\left(x_{0}, x_{1}\right)\right)<\varepsilon .
$$

This implies that $\left\{x_{n}\right\}$ is a Cauchy sequence in $X$. By completeness of $X$, there exists $x \in X$ such that $x_{n} \rightarrow x$ as $n \rightarrow \infty$.

Finally, we show that $p_{\alpha}(x, T x)=0$. Assume on the contrary that $p_{\alpha}(x, T x)>0$. By condition (iii), we have $\beta\left(x_{n}, x\right) \geq 1$ for all $n \in \mathbb{N}$. Now we have

$$
\begin{aligned}
p_{\alpha}(x, T x) \leq & d\left(x_{n}, x_{n+1}\right)+p_{\alpha}\left(x_{n+1}, T x\right) \\
\leq & d\left(x_{n}, x_{n+1}\right)+D_{\alpha}\left(T x_{n}, T x\right) \\
\leq & d\left(x_{n}, x_{n+1}\right)+\beta\left(x_{n}, x\right) D_{\alpha}\left(T x_{n}, T x\right) \\
\leq & d\left(x_{n}, x_{n+1}\right)+\psi\left(\max \left\{d\left(x_{n}, x\right), p_{\alpha}\left(x_{n}, T x_{n}\right), p_{\alpha}(x, T x), \frac{p_{\alpha}\left(x_{n}, T x\right)+p_{\alpha}\left(x, T x_{n}\right)}{2}\right\}\right) \\
& +L \min \left\{p_{\alpha}\left(x_{n}, T x_{n}\right), p_{\alpha}(x, T x), p_{\alpha}\left(x_{n}, T x\right), p_{\alpha}\left(x, T x_{n}\right)\right\} \\
\leq & d\left(x_{n}, x_{n+1}\right)+\psi\left(\max \left\{d\left(x_{n}, x\right), d\left(x_{n}, x_{n+1}\right), p_{\alpha}(x, T x), \frac{p_{\alpha}\left(x_{n}, T x\right)+d\left(x, x_{n+1}\right)}{2}\right\}\right) \\
& +L \min \left\{p_{\alpha}\left(x_{n}, x_{n+1}\right), p_{\alpha}(x, T x), p_{\alpha}\left(x_{n}, T x\right), p_{\alpha}\left(x, T x_{n}\right)\right\} .
\end{aligned}
$$


Letting $n \rightarrow \infty$, it follows that

$$
p_{\alpha}(x, T x) \leq \psi\left(p_{\alpha}(x, T x)\right)<p_{\alpha}(x, T x),
$$

which is a contradiction. Therefore, we have $p_{\alpha}(x, T x)=0$. Hence, by Lemma $1, x_{\alpha} \subset T x$. This complete the proof.

Next, we give some examples to support the validity of our result.

Example 2. Let $X=[0,1]$ and $d: X \times X \rightarrow[0, \infty)$ as $d(x, y)=|x-y|$ for all $x, y \in X$. Then $(X, d)$ is a complete metric space. Let us define $T: X \rightarrow I^{X}$ by

$$
(T x)(t)=\left\{\begin{array}{l}
0,0 \leq t<\frac{1}{6}, \\
\frac{3}{4}, \frac{1}{6} \leq t \leq \frac{x+1}{6}, \\
\frac{1}{2}, \frac{x+1}{6}<t \leq 1 .
\end{array}\right.
$$

Let $\alpha=\frac{3}{4}$. We observe that

$$
[T x]_{\alpha}=[T x]_{\frac{3}{4}}=\left[\frac{1}{6}, \frac{x+1}{6}\right]
$$

for all $x \in X$. Therefore, $T$ is fuzzy mapping from $X$ to $W_{\alpha}(X)$.

Define $\beta: X \times X \rightarrow[0, \infty)$ by $\beta(x, y)=2$ for all $x, y \in X$. Then it is easy to check that $T$ is an $\beta$-admissible. For each $x, y \in X$, we get

$$
\begin{aligned}
\beta(x, y) D_{\alpha}(T x, T y) & =\beta(x, y) H\left([T x]_{\alpha},[T y]_{\alpha}\right) \\
& =\frac{1}{3}|x-y| \\
& =\frac{1}{3} d(x, y) \\
& <\frac{1}{2} d(x, y) \\
& \leq \psi(M(x, y))+L \min \left\{p_{\alpha}(x, T x), p_{\alpha}(y, T y), p_{\alpha}(x, T y), p_{\alpha}(y, T x)\right\},
\end{aligned}
$$

where $\psi(t)=\frac{t}{2}$ for all $t>0$ and $L \geq 0$. It is easy to see that conditions (ii) and (iii) in Theorem 1 hold. Therefore, all conditions of Theorem 1 hold. Thus, $T$ has an $\alpha$-fuzzy fixed point $x \in X$, that is, a point $x=\frac{1}{6}$.

By using Remark 1, we get the following result.

Theorem 2. Let $(X, d)$ be a complete metric space, $\alpha \in[0,1]$ and $T$ be fuzzy mapping from $X$ to $W_{\alpha}(X)$. Suppose that there exist $\psi \in \Psi$ and $\beta: X \times X \rightarrow[0, \infty)$ such that

$$
\beta(x, y) D_{\alpha}(T x, T y) \leq \psi(M(x, y))+L \min \left\{p_{\alpha}(x, T x), p_{\alpha}(y, T y), p_{\alpha}(x, T y), p_{\alpha}(y, T x)\right\}
$$

for all $x, y \in X$, where $L \geq 0$ and

$$
M(x, y)=\max \left\{d(x, y), p_{\alpha}(x, T x), p_{\alpha}(y, T y), \frac{p_{\alpha}(x, T y)+p_{\alpha}(y, T x)}{2}\right\} .
$$


If the following condition holds,

(i) $T$ is $\beta^{*}$-admissible,

(ii) there exist $x_{0} \in X$ and $x_{1} \in\left[T x_{0}\right]_{\alpha}$ such that $\beta\left(x_{0}, x_{1}\right) \geq 1$,

(iii) if $\left\{x_{n}\right\}$ is sequence in $X$ such that $\beta\left(x_{n}, x_{n+1}\right) \geq 1$ and $x_{n} \rightarrow u$ as $n \rightarrow \infty$, then $\beta\left(x_{n}, u\right) \geq 1$,

(iv) $\psi$ is continuous,

then there exists $x \in X$ such that $x_{\alpha}$ is a fuzzy fixed point of $T$.

In Theorems 1 and 2 , we take $\psi(t)=\theta t$, where $\theta \in(0,1)$ then we have the following corollary which is a fuzzy extension of fixed point theorem given by Berinde [16].

Corollary 1. Let $(X, d)$ be a complete metric space, $\alpha \in[0,1]$ and $T$ be fuzzy mapping from $X$ to $W_{\alpha}(X)$. Suppose that there exists $\beta: X \times X \rightarrow[0, \infty)$ such that

$$
\beta(x, y) D_{\alpha}(T x, T y) \leq \theta M(x, y)+L \min \left\{p_{\alpha}(x, T x), p_{\alpha}(y, T y), p_{\alpha}(x, T y), p_{\alpha}(y, T x)\right\}
$$

for all $x, y \in X$, where, $\theta \in(0,1), L \geq 0$ and

$$
M(x, y)=\max \left\{d(x, y), p_{\alpha}(x, T x), p_{\alpha}(y, T y), \frac{p_{\alpha}(x, T y)+p_{\alpha}(y, T x)}{2}\right\} .
$$

If the following condition holds,

(i) $\quad T$ is $\beta$-admissible (or $\beta^{*}$-admissible),

(ii) there exist $x_{0} \in X$ and $x_{1} \in\left[T x_{0}\right]_{\alpha}$ such that $\beta\left(x_{0}, x_{1}\right) \geq 1$,

(iii) if $\left\{x_{n}\right\}$ is sequence in $X$ such that $\beta\left(x_{n}, x_{n+1}\right) \geq 1$ and $x_{n} \rightarrow u$ as $n \rightarrow \infty$, then $\beta\left(x_{n}, u\right) \geq 1$,

then there exists $x \in X$ such that $x_{\alpha}$ is a fuzzy fixed point of $T$.

If we take $L=0$ in Theorems 1 and 2 and Corollary 1 , then we have the following corollaries:

Corollary 2. Let $(X, d)$ be a complete metric space, $\alpha \in[0,1]$ and $T$ be fuzzy mapping from $X$ to $W_{\alpha}(X)$. Suppose that there exist $\psi \in \Psi$ and $\beta: X \times X \rightarrow[0, \infty)$ such that

$$
\beta(x, y) D_{\alpha}(T x, T y) \leq \psi(M(x, y))
$$

for all $x, y \in X$ and

$$
M(x, y)=\max \left\{d(x, y), p_{\alpha}(x, T x), p_{\alpha}(y, T y), \frac{p_{\alpha}(x, T y)+p_{\alpha}(y, T x)}{2}\right\} .
$$

If the following condition holds,

(i) $\quad T$ is $\beta$-admissible (or $\beta^{*}$-admissible),

(ii) there exist $x_{0} \in X$ and $x_{1} \in\left[T x_{0}\right]_{\alpha}$ such that $\beta\left(x_{0}, x_{1}\right) \geq 1$,

(iii) if $\left\{x_{n}\right\}$ is sequence in $X$ such that $\beta\left(x_{n}, x_{n+1}\right) \geq 1$ and $x_{n} \rightarrow u$ as $n \rightarrow \infty$, then $\beta\left(x_{n}, u\right) \geq 1$,

(iv) $\psi$ is continuous,

then there exists $x \in X$ such that $x_{\alpha}$ is a fuzzy fixed point of $T$. 
Corollary 3. Let $(X, d)$ be a complete metric space, $\alpha \in[0,1]$ and $T$ be fuzzy mapping from $X$ to $W_{\alpha}(X)$. Suppose that there exists $\beta: X \times X \rightarrow[0, \infty)$ such that

$$
\beta(x, y) D_{\alpha}(T x, T y) \leq \theta \max \left\{d(x, y), p_{\alpha}(x, T x), p_{\alpha}(y, T y), \frac{p_{\alpha}(x, T y)+p_{\alpha}(y, T x)}{2}\right\}
$$

for all $x, y \in X$, where $\theta \in(0,1)$. If the following condition holds,

(i) $T$ is $\beta$-admissible (or $\beta^{*}$-admissible),

(ii) there exist $x_{0} \in X$ and $x_{1} \in\left[T x_{0}\right]_{\alpha}$ such that $\beta\left(x_{0}, x_{1}\right) \geq 1$,

(iii) if $\left\{x_{n}\right\}$ is sequence in $X$ such that $\beta\left(x_{n}, x_{n+1}\right) \geq 1$ and $x_{n} \rightarrow u$ as $n \rightarrow \infty$, then $\beta\left(x_{n}, u\right) \geq 1$,

then there exists $x \in X$ such that $x_{\alpha}$ is a fuzzy fixed point of $T$.

If we set $\beta(x, y)=1$ for all $x, y \in X$ in Theorem 1 or Theorem 2, we get the following result:

Corollary 4. Let $(X, d)$ be a complete metric space, $\alpha \in[0,1]$ and $T$ be fuzzy mapping from $X$ to $W_{\alpha}(X)$. Suppose that there exist $\psi \in \Psi$ such that

$$
D_{\alpha}(T x, T y) \leq \psi(M(x, y))+L \min \left\{p_{\alpha}(x, T x), p_{\alpha}(y, T y), p_{\alpha}(x, T y), p_{\alpha}(y, T x)\right\}
$$

for all $x, y \in X$, where $L \geq 0$ and

$$
M(x, y)=\max \left\{d(x, y), p_{\alpha}(x, T x), p_{\alpha}(y, T y), \frac{p_{\alpha}(x, T y)+p_{\alpha}(y, T x)}{2}\right\} .
$$

Then there exists $x \in X$ such that $x_{\alpha}$ is a fuzzy fixed point of $T$.

If $\psi(t)=\theta t$, where $\theta \in(0,1)$ and $\beta(x, y)=1$ for all $x, y \in X$ in Theorem 1 or Theorem 2 , then we have the following corollary.

Corollary 5. Let $(X, d)$ be a complete metric linear space, $\alpha \in[0,1]$ and $T$ be fuzzy mapping from $X$ to $W_{\alpha}(X)$ such that

$$
D_{\alpha}(T x, T y) \leq \theta \max \left\{d(x, y), p_{\alpha}(x, T x), p_{\alpha}(y, T y), \frac{p_{\alpha}(x, T y)+p_{\alpha}(y, T x)}{2}\right\}
$$

for all $x, y \in X$, where $\theta \in(0,1)$. Then there exists $x \in X$ such that $x_{\alpha}$ is a fuzzy fixed point of $T$.

\section{Applications}

In this section, we give the existence of fuzzy fixed point theorems in a partially ordered metric space.

Before presenting our results, we give the following definitions.

Definition 8. Let $X$ be a nonempty set. Then $(X, d, \preceq)$ is said to be an ordered metric spaces if $(X, d)$ is a metric space and $(X, \preceq)$ is a partially ordered set.

Definition 9. Let $(X, \preceq)$ be a partially ordered set. Then $x, y \in X$ are said to be comparable if $x \preceq y$ or $y \preceq x$ holds. 
For partially ordered set $(X, \preceq)$, we define

$$
\bar{\wedge}:=\{(x, y) \in X \times X: x \preceq y \text { or } y \preceq x\} .
$$

Definition 10. A partially ordered set $(X, \preceq)$ is said to satisfy the order sequential limit property if $\left(x_{n}, x\right) \in \bar{\wedge}$ for all $n \in \mathbb{N}$, whenever a sequence $x_{n} \rightarrow x$ as $n \rightarrow \infty$ and $\left(x_{n}, x_{n+1}\right) \in \bar{\wedge}$ for all $n \in \mathbb{N}$.

Example 3. Let $X=\mathbb{R}$ and $\preceq=\leq$ be a partial ordered on $X$. It is easy to check that $(X, d, \preceq)$ satisfy the order sequential limit property.

Definition 11. Let $(X, \preceq)$ be a partially ordered set and $\alpha \in[0,1]$. A fuzzy mapping $T: X \rightarrow W_{\alpha}(X)$ is said to be comparative if for each $x \in X$ and $y \in[T x]_{\alpha}$, with $(x, y) \in \bar{\wedge}$, we have $(y, z) \in \bar{\wedge}$ for all $z \in[T y]_{\alpha}$.

Here we give the existence of fuzzy fixed point theorem for fuzzy mapping in ordered metric spaces.

Theorem 3. Let $(X, d, \preceq)$ be a complete partially ordered metric space, $\alpha \in[0,1]$ and $T$ be fuzzy mapping from $X$ to $W_{\alpha}(X)$. Suppose that there exists $\psi \in \Psi$ such that

$$
D_{\alpha}(T x, T y) \leq \psi(M(x, y))+L \min \left\{p_{\alpha}(x, T x), p_{\alpha}(y, T y), p_{\alpha}(x, T y), p_{\alpha}(y, T x)\right\}
$$

for all $(x, y) \in \bar{\wedge}$, where $L \geq 0$ and

$$
M(x, y)=\max \left\{d(x, y), p_{\alpha}(x, T x), p_{\alpha}(y, T y), \frac{p_{\alpha}(x, T y)+p_{\alpha}(y, T x)}{2}\right\} .
$$

If the following condition holds,

(I) $T$ is comparative fuzzy mapping,

(II) there exist $x_{0} \in X$ and $x_{1} \in\left[T x_{0}\right]_{\alpha}$ such that $\left(x_{0}, x_{1}\right) \in \bar{\wedge}$,

(III) $X$ satisfies the order sequential limit property,

(IV) $\psi$ is continuous,

then there exists $x \in X$ such that $x_{\alpha}$ is a fuzzy fixed point of $T$.

Proof. Consider the mapping $\beta: X \times X \rightarrow[0, \infty)$ defined by

$$
\beta(x, y)=\left\{\begin{array}{l}
1 \text { if }(x, y) \in \bar{\wedge}, \\
0 \text { if }(x, y) \notin \bar{\wedge} .
\end{array}\right.
$$

From condition (II), we get $\beta\left(x_{0}, x_{1}\right) \geq 1$ and then the condition (ii) in Theorem 1 holds. It follows from $T$ is comparative fuzzy mapping that $T$ is $\beta$-admissible mapping. By (18), we have, for all $x, y \in X$,

$$
\beta(x, y) D_{\alpha}(T x, T y) \leq \psi(M(x, y))+L \min \left\{p_{\alpha}(x, T x), p_{\alpha}(y, T y), p_{\alpha}(x, T y), p_{\alpha}(y, T x)\right\} .
$$

Since $X$ satisfies the order sequential limit property, the condition (iii) in Theorem 1 is also holds. Now all the hypotheses of Theorem 1 are satisfied and thus the existence of the fuzzy fixed point of fuzzy mapping $T$ follows from Theorem 1. 
By using the same technique in the proof of Theorem 3 with Corollaries 1, 2 and 3, we get the following results.

Corollary 6. Let $(X, d, \preceq)$ be a complete partially ordered metric space, $\alpha \in[0,1]$ and $T$ be fuzzy mapping from $X$ to $W_{\alpha}(X)$ such that

$$
D_{\alpha}(T x, T y) \leq \theta M(x, y)+L \min \left\{p_{\alpha}(x, T x), p_{\alpha}(y, T y), p_{\alpha}(x, T y), p_{\alpha}(y, T x)\right\}
$$

for all $(x, y) \in \bar{\wedge}$, where $L \geq 0$ and

$$
M(x, y)=\max \left\{d(x, y), p_{\alpha}(x, T x), p_{\alpha}(y, T y), \frac{p_{\alpha}(x, T y)+p_{\alpha}(y, T x)}{2}\right\} .
$$

If the following condition holds,

(I) $T$ is comparative fuzzy mapping,

(II) there exist $x_{0} \in X$ and $x_{1} \in\left[T x_{0}\right]_{\alpha}$ such that $\left(x_{0}, x_{1}\right) \in \bar{\wedge}$,

(III) $X$ satisfies the order sequential limit property,

then there exists $x \in X$ such that $x_{\alpha}$ is a fuzzy fixed point of $T$.

Corollary 7 . Let $(X, d, \preceq)$ be a complete partially ordered metric space, $\alpha \in[0,1]$ and $T$ be fuzzy mapping from $X$ to $W_{\alpha}(X)$. Suppose that there exists $\psi \in \Psi$ such that

$$
D_{\alpha}(T x, T y) \leq \psi(M(x, y))
$$

for all $(x, y) \in \bar{\wedge}$, where $L \geq 0$ and

$$
M(x, y)=\max \left\{d(x, y), p_{\alpha}(x, T x), p_{\alpha}(y, T y), \frac{p_{\alpha}(x, T y)+p_{\alpha}(y, T x)}{2}\right\} .
$$

If the following condition holds,

(I) $T$ is comparative fuzzy mapping,

(II) there exist $x_{0} \in X$ and $x_{1} \in\left[T x_{0}\right]_{\alpha}$ such that $\left(x_{0}, x_{1}\right) \in \bar{\wedge}$,

(III) $X$ satisfies the order sequential limit property,

(IV) $\psi$ is continuous,

then there exists $x \in X$ such that $x_{\alpha}$ is a fuzzy fixed point of $T$.

Corollary 8. Let $(X, d, \preceq)$ be a complete partially ordered metric space, $\alpha \in[0,1]$ and $T$ be fuzzy mapping from $X$ to $W_{\alpha}(X)$ such that

$$
D_{\alpha}(T x, T y) \leq \theta M(x, y)
$$

for all $(x, y) \in \bar{\wedge}$, where

$$
M(x, y)=\max \left\{d(x, y), p_{\alpha}(x, T x), p_{\alpha}(y, T y), \frac{p_{\alpha}(x, T y)+p_{\alpha}(y, T x)}{2}\right\} .
$$

If the following condition holds,

(I) $T$ is comparative fuzzy mapping,

(II) there exist $x_{0} \in X$ and $x_{1} \in\left[T x_{0}\right]_{\alpha}$ such that $\left(x_{0}, x_{1}\right) \in \bar{\wedge}$,

(III) $X$ satisfies the order sequential limit property,

then there exists $x \in X$ such that $x_{\alpha}$ is a fuzzy fixed point of $T$. 


\section{Acknowledgements}

The second authors gratefully acknowledge the financial support provided by Thammasat University under the TU Research Scholar, Contract No. 2/10/2557.

\section{Author details}

${ }^{1}$ Department of Mathematics, Faculty of Science, King Mongkut's University of Technology Thonburi (KMUTT), 126 Pracha Uthit Rd., Bang Mod, Thung Khru, Bangkok 10140, Thailand. ${ }^{2}$ Department of Mathematics and Statistics, Faculty of Science and Technology, Thammasat University Rangsit Center, Pathumthani 12121, Thailand.

Received: 21 May 2014 Accepted: 17 August 2014

Published online: 06 September 2014

\section{References}

1. Zadeh, LA: Fuzzy sets. Inf. Control. 8, 103-112 (1965)

2. Heilpern, S: Fuzzy mappings and fixed point theorems. J. Math. Anal. Appl. 83, 566-569 (1981)

3. Nadler, SB Jr: Multivalued contraction mapping. Pac. J. Math. 30(2), 475-488 (1969)

4. Estruch, VD, Vidal, A: A note on fixed fuzzy points for fuzzy mappings. Rend Istit. Univ. Trieste. 32, 39-45 (2001)

5. Azam, A, Beg, l: Common fixed points of fuzzy maps. Math. Comput. Model. 49, 1331-1336 (2009)

6. Turkoglu, D, Rhoades, BE: A fixed fuzzy point for fuzzy mapping in complete metric spaces. Math. Commun. 10, 115-121 (2005)

7. Sedghi, S, Shobe, N, Altun, I: A fixed fuzzy point for fuzzy mappings in complete metric spaces. Math. Commun. 13, 289-294 (2008)

8. Phiangsungnoen, $S$, Sintunavarat, $W$, Kumam, P: Common $\alpha$-fuzzy fixed point theorems for fuzzy mappings via $\beta_{\mathcal{F}}$-admissible pair. J. Intell. Fuzzy Syst (2014). doi.org/10.3233/IFS-141218

9. Phiangsungnoen, S, Sintunavarat, W, Kumam, P: Fuzzy fixed point theorems in Hausdorff fuzzy metric spaces. J. Inequalities Appl. 2014, 201 (2014)

10. Abbas, M, Turkoglu, D: Fixed point theorem for a generalized contractive fuzzy mapping. J. Intell. Fuzzy Syst. 26(1), 33-36 (2014)

11. Samet, B, Vetro, C, Vetro, P: Fixed point theorems for $\alpha$ - $\psi$-contractive type mappings. Nonlinear Anal. 75, 2154-2165 (2012)

12. Asl, JH, Rezapour, S, Shahzad, N: On fixed points of $\alpha-\psi$-contractive multifunctions. Fixed Point Theory Appl. 2012, 212 (2012)

13. Mohammadi, B, Rezapour, S, Shahzad, N: Some results on fixed points of $\alpha$ - $\psi$-Ciric generalized multifunctions. Fixed Point Theory Appl. 2013, 24 (2013)

14. Azam, A, Hussain, S, Arshad, M: Common fixed points of Chatterjea type fuzzy mappings on closed balls. Neural Comput. Appl. 21(1 Supplement), 313-317 (2012)

15. Pu, P-M, Liu, Y-M: Fuzzy topology. I. Neighborhood structure of a fuzzy point and Moore-smith convergence. J. Math. Anal. Appl. 76, 571-599 (1980)

16. Berinde, V: General constructive fixed point theorems for Ćirić- type almost contractions in metric spaces. Carpathian J. Math. 24(2), 10-19 (2008)

\section{doi:10.1186/s40467-014-0020-6}

Cite this article as: Phiangsungnoen et al:: Fuzzy fixed point theorems for fuzzy mappings via $\beta$-admissible with applications. Journal of Uncertainty Analysis and Applications 2014 2:20.

\section{Submit your manuscript to a SpringerOpen ${ }^{\circ}$ journal and benefit from:}

- Convenient online submission

Rigorous peer review

- Immediate publication on acceptance

- Open access: articles freely available online

- High visibility within the field

- Retaining the copyright to your article

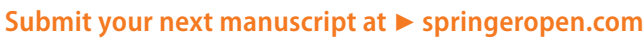

